\title{
DIROFILARIOSE CANINA: REVISÃO DE UMA ZOONOSE EMERGENTE
}

\section{Canine Dirofilariosis: a Revision about an Emerging Zoonosis}

\author{
Luiz Carlos Leite \\ Silvana Maris Cirio ${ }^{2}$ \\ Valter da Silva Queiroz ${ }^{3}$ \\ Mario Antonio Navarro da Silva ${ }^{4}$ \\ Ennio $\mathrm{Luz}^{5}$ \\ Herminio de Paula Molinari ${ }^{6}$ \\ João Maria Ferraz Diniz \\ Selene Cirio Leite \\ Diego Lunelli ${ }^{9}$ \\ Stefany Weber ${ }^{10}$ \\ Ana Carolina Bazo Zadorosnei ${ }^{11}$
}

\section{Resumo}

O nematóide Dirofilaria immitis (Leidy, 1856) (Spirurida: Oncocercidae) é um parasita encontrado no ventrículo direito e artéria pulmonar de carnívoros domésticos e selvagens e de outros animais, como coelhos e cavalos. As lesões causam problemas cardiopulmonares severos e a morte de alguns animais. Esta revisão abrange os aspectos gerais, ciclo biológico, vetores potenciais, prevenção e controle da Dirofilaria immitis.

Palavras-chave: Dirofilaria immitis; Infecção; Dirofilariose.

1 Prof. Adjunto, Curso de Medicina Veterinária, UNIGUAÇU-PR, Travessa Ângelo Piazzetta - 101, Cristo Rei - Curitiba - Paraná, CEP 80050-460 e-mail: "mailto:tudolica@pop.com.br" tudolica@pop.com.br

2 Prof. ${ }^{a}$ Adjunta, Curso de Medicina Veterinária, PUCPR, Travessa Ângelo Piazzetta - 101, Cristo Rei - Curitiba - Paraná, CEP 80050460 e-mail: tudolica@pop.com.br

3 Prof. Adjunto, Curso de Medicina Veterinária, PUCPR, Rua José de Mello Braga Jr. , n. 65, Jardim das Américas Curitiba - Paraná, CEP 81540-280, e-mail: valterqueiroz@pucpr.br

4 Prof. Adjunto, Departamento de Entomologia, UFPR, Rua Martim Afonso, 2915 Ap. 63, Campina do Siqueira - Curitiba - Paraná, CEP 80730-030, e-mail: pgento@bio.ufpr.br

5 Prof. Titular, Departamento de Patologia Básica, UFPR, Rua Paula Gomes, 766 Ap. 101, Curitiba - Paraná, CEP 80510-070, e-mail: mariliatardeli@hotmail.com

6 Prof. Auxiliar de Ensino, Curso de Medicina Veterinária, PUCPR, Rodovia BR 376 - km 14 - Costeira - São José dos Pinhais, email: herminiomolinari@gmail.com

7 Prof. Adjunto Aposentado, Departamento de Medicina Veterinária, UFPR, Rua São Tomé - 58, Vila Santa Cruz, Castro- Paraná, CEP 84188-010

8 Acadêmica, Curso de Medicina Veterinária, PUCPR, Travessa Ângelo Piazzetta - 101, Cristo Rei - Curitiba - Paraná, CEP 80050460 e-mail: tudolica@pop.com.br

9 Acadêmico, Curso de Medicina Veterinária, PUCPR, Travessa Ângelo Piazzetta - 101, Cristo Rei - Curitiba - Paraná, CEP 80050460 e-mail: tudolica@pop.com.br

10 Acadêmica, Curso de Medicina Veterinária, PUCPR, Rua Manoel Correia de Freitas - 727, Jardim Social - Curitiba - Paraná, CEP 82530-070, e-mail: stefanyweber@yahoo.com.br

11 Acadêmica, Curso de Medicina Veterinária, PUCPR, Rua Ary Barroso - 827, Boa Vista - Curitiba - Paraná, CEP 82540-000, e-mail: ana_bazo@brturbo.com 


\section{Abstract}

Dirofilaria immitis (Leidy, 1856) ( Spirurida: Oncocercidae) is a parasite nematode that infects the right ventricle and pulmonary artery of domestic and wild carnivores, and other animals like rabbits and horses. The lesions cause severe cardiopulmonary problems and death in some animals. This review refers the general aspects, biological cycle, potential vectors, prevention and control of Dirofilaria immitis.

Keywords: Dirofilaria immitis; Infection; Dirofilariasis.

\section{Introdução}

Conforme demonstrado em estudos por Russel (1985); Loftin (1995); Junkum et al. (2003), a Dirofilaria immitis (Leidy, 1856) Raillet \& Henry (1911) é transmitida por mosquitos (Diptera: Culicidae) e ocorre com acentuada prevalência em áreas litorâneas de países localizados nos cinco continentes. No Brasil, existem registros do parasita em regiões com diferentes graus de incidência (KASAI et al., 1981; AHID et al., 1999; ARAUJO et al., 2003; SUASSUANA et al., 2003; COSTA et al., 2004; REIFUR et al., 2004).

O primeiro pesquisador a relatar a presença de Dirofilaria immitis em um cão foi Osborne (1847). Descrita nos Estados Unidos em 1856, inicialmente registrada como Filaria immitis (LEIDY, 1856). Mais tarde foi reclassificada no gênero Dirofilaria (RAILLET; HENRY, 1911). De acordo com Pinto; Luz (1936), o primeiro registro de $D i$ rofilaria immitis no Brasil foi de Silva Araujo (1878). Travassos (1921) relatou pela primeira vez em nosso país a doença em gatos.

As fêmeas e machos adultos parasitam o ventrículo direito e freqüentemente o tronco principal das artérias pulmonares de canídeos e outras espécies de animais domésticos e silvestres, determinando endarterite, alterações no fluxo sanguíneo e na constituição do sangue. As microfilárias, larvas de primeiro instar, medem em torno de 315 $\mu \mathrm{m}(295-325 \mu \mathrm{m})$ de comprimento e 6 a $7 \mu \mathrm{m}(5,0-$ $7,5 \mu \mathrm{m})$ de diâmetro. Detectadas na circulação periférica de cães, quando submetidas à Técnica de KNOTT modificada por Newton; Wright (1956), apresentam o corpo com a extremidade cefálica afilada e extremidade caudal distendida, sobrevivem na corrente circulatória por um período variável de até dois anos, estando presentes em 60\% dos cães portadores de dirofilariose (LINDSEY, 1965; ETTINGER; FELDMAN, 2004). Os parasitas adultos são longos, de coloração esbranquiçada, com acentuado dimorfismo sexual. Machos medem de 12 a $20 \mathrm{~cm}$ de comprimento com cauda espiralada, enquanto as fêmeas medem entre 25 e $31 \mathrm{~cm}$ de comprimento, apresentando extremidade caudal arredondada.

O ciclo de vida inclui a passagem obrigatória das microfilárias por hospedeiros intermediários, que são mosquitos culicídeos suscetíveis (LUDLAM et al., 1970). Esses se infectam ao alimentar-se em animais microfilarêmicos, considerados como o mais importante fator que contribui para a disseminação do parasita (McCALL et al., 2004). Após a ingestão pelo mosquito, num prazo entre 24 e 36 horas, as microfilárias dirigem-se do intestino aos túbulos de Malpighi, onde completam o desenvolvimento embrionário em torno de 10 a 30 dias e depois de dois estádios evolutivos até a fase larval infectante $\mathrm{L}^{3}$ migram pelo tórax em sentido cranial, retornando para as peças bucais (probóscide) do mosquito. Por ocasião de um novo repasto sangüíneo, são transmitidas aos hospedeiros definitivos, penetrando no orifício resultante da picada, pela pele íntegra ou ainda pelos folículos pilosos, onde migrarão ativamente pelos músculos e tecidos subcutâneo e adiposo, realizando mudas para as fases larvais L ${ }^{4}$ e $\mathrm{L}^{5}$ (OTTO, 1969). Quando atingem em torno de $5 \mathrm{~cm}$ de comprimento, dirigem-se ao ventrículo direito. As formas imaturas chegam neste local entre 70 e 90 dias após o início da infecção (ORIHEL, 1961; KOTANI; POWERS, 1982). Em torno de seis a oito meses, os parasitas atingem a maturidade sexual e iniciam a reprodução. As fêmeas adultas são vivíparas e produzem as microfilárias $\mathrm{L}^{1}$, que são liberadas diretamente na circulação do hospedeiro definitivo, completando o ciclo do parasita.

Os nematóides adultos produzem lesões endoteliais, tromboembolismo, hipertensão pulmonar, pneumonite, congestão hepática, ascite e glomerulonefrite como conseqüência da deposição de imunocomplexos nos glomérulos (PAES-DE-ALMEIDA et al., 2003). As alterações patológicas no sistema cardiovascular do hospedeiro definitivo ocorrem em conseqüência de lesões provocadas pela ação de parasitas adultos, microfilárias e larvas 
migratórias jovens. A localização primária dá-se freqüentemente nas artérias pulmonares de animais com baixo número de parasitas. Quando a carga parasitária é alta, existe migração ativa desde as artérias pulmonares até o ventrículo e átrio direitos e com menor intensidade até a veia cava. Dirofilárias adultas podem ser observadas ao redor da válvula tricúspide ou da cordoalha tendinosa do coração (SCHREY; TRAUTVETTER, 1998).

Sinais moderados da doença incluem intolerância a exercícios físicos, tosse crônica, queda do apetite e perda de peso progressiva, enquanto a fase grave compreende características como taquipnéia, falência congestiva do lado direito do coração, ascite, esplenomegalia, hepatomegalia, edema subcutâneo, tromboembolismo pulmonar e síndrome caval aguda ou crônica (STRICKLAND, 1998).

Síndrome Caval, também descrita como Síndrome da Veia Cava, Síndrome Pós-Caval, Síndrome Hepática Aguda, Embolismo da Veia Cava ou Hemoglobinúria Dirofilarial, é uma séria complicação registrada igualmente nos cães e gatos portadores de dirofilariose crônica. Os achados clínicos podem incluir tosse, dispnéia, letargia, taquicardia, anorexia acentuada, taquipnéia, ascite, distensão e pulsação da veia jugular, anemia, hemoglobinemia, disfunção renal e hepática, hemoglobinúria e coagulação intravascular disseminada (CID). A causa é a movimentação de grande número de dirofilárias adultas das artérias pulmonares danificadas para o ventrículo e átrio direitos e/ou veia cava, resultando em regurgitação moderada ou severa observada na válvula tricúspide e falência cardíaca. As cargas parasitárias observadas são geralmente altas. Entretanto, alguns casos de síndrome caval já foram registrados em cães com baixa carga parasitária (HIDAKA et al., 2003).

O diagnóstico clínico da dirofilariose inclui a avaliação de sinais e sintomas apresentados pelo animal suspeito como intolerância a exercícios físicos, tosse, emagrecimento, debilidade e dispnéia. A presença de microfilárias na circulação periférica é importante no estabelecimento do diagnóstico da doença. O número de microfilárias normalmente não tem relação direta com a presença de filárias adultas e também com a severidade da doença. A microfilaremia pode ser detectada mesmo após a eliminação dos parasitas adultos.
Filhotes nascidos de cadelas que albergam alta porcentagem de microfilárias podem infectar-se via transplacentária e apresentar uma microfilaremia de caráter transitório que não resultará em infecção por parasitas adultos e normalmente desaparece após dois meses (MANTOVANI; JACKSON, 1966; ETTINGER; FELDMAN, 2004).

A dirofilariose oculta, caracterizada por ausência de microfilaremia, pode ocorrer pela destruição das microfilárias por meio de mecanismos imunomediados no período pré-patente da doença, vermes imaturos, tratamento com drogas apenas microfilaricidas, morte das formas adultas ou ainda pela presença apenas de parasitas machos ou fêmeas (KELLY, 1973).

Enquanto o cão é infectado mais comumente enquadrando-se como reservatório significativo, o gato é considerado hospedeiro atípico de Dirofilaria immitis (ATKINS et al., 2000; BROWNE et al., 2005). A dirofilariose felina, embora ocorrendo de maneira menos freqüente que a canina mesmo em áreas endêmicas, também é de distribuição geográfica cosmopolita. Os sinais clínicos da doença em gatos incluem dispnéia, tosse, vômito, perda de peso e hipertrofia severa ou média das paredes da artéria pulmonar, sendo o diagnóstico dificultado por baixa carga de parasitas e rara microfilaremia (GOODFELOW; SHAW, 2005; BROWNE et al., 2005). Em cães e gatos, além da localização no coração e artérias pulmonares, exemplares adultos de Dirofilaria immitis podem ser encontrados ocasionalmente em infecções ectópicas localizadas fora do sistema vascular, em áreas como bronquíolos, tecido conjuntivo subcutâneo, câmara anterior do olho e sistema nervoso central (HAMIR, 1987). Por meio de migração somática aberrante, as larvas se localizam nestes sítios e aí podem evoluir até o estado adulto (SCHREY; TRAUTVETTER, 1998). Já as microfilárias normalmente estão presentes na circulação sanguínea periférica destes animais (KELLY, 1973; HAYASAKI et al., 2003).

\section{Dirofilaria immitis EM OUTROS ANIMAIS}

Considerável casuística foi registrada em outras espécies animais. O parasitismo envolve leões marinhos (FORRESTER et al., 1973), castores (FOIL; ORHIEL, 1975), carcajou (WILLIAMS; DADE, 1976), coiotes (FRANSON et al., 1976), urso negro 
(JOHNSON, 1975), cavalos (KLEIN; STODDARD, 1977; THURMAN et al., 1984), urso panda vermelho (HARWELL; CRAIG, 1981), raposa cinzenta (CARLSON; NIELSEN, 1983), dingos (STARR; MULLEY, 1988), guaxinins (SNYDER et al., 1989a), lontras (SNYDER et al., 1989b; MATSUDA et al., 2003; TORRES et al., 2004), coelhos (NAKAGAKI et al., 1997), lobo marinho sul-americano (MARANHO et al., 1997), raposas vermelhas (GORTAZAR et al., 1994; MARKS; BLOOMFIELD, 1998), leões e tigres (GUPTA et al., 1999), furão (SASAI et al., 2000), lobos (SEGOVIA et al., 2001), foca (KANG et al., 2002) e jaguatiricas (PENCE et al., 2003).

\section{DIROFILARIOSE EM SERES HUMANOS}

Em seres humanos Dirofilaria immitis, é geralmente encontrada em forma imatura no interior de nódulos localizados nos pulmões, sendo freqüentemente confundido com neoplasia (NARINE et al., 1999). Os parasitas raramente atingem a fase adulta e as formas imaturas que morrem no coração são carreadas para os pulmões pela artéria pulmonar, podendo produzir nódulos e sintomas de tromboembolismo, causando dirofilariose pulmonar humana (MILANEZ DE CAMPOS et al., 1997; SHAH, 1999; OSHIRO et al., 2004; RODRIGUES-SILVA et al., 2004; VITAL et al., 2006). Igualmente foram descritas localizações em cavidade abdominal (TADA et al., 1979), coração e veia cava anterior (TAKEUCHI et al., 1981), cordões espermáticos (THEIS et al., 2001) e tecidos periorbital (RODRIGUEZ et al., 2003) e hepático (KIM et al., 2002).

\section{DISTRIBUIÇÃO GEOGRÁFICA DA DIROFILARIOSE}

A dirofilariose é endêmica nos países do sul da Europa, destacando-se em Portugal, Espanha, Grécia (POLIZOPOULOU et al., 2000), sul da França e Itália, nas Américas, Oceania (MARKS; BLOOMFIELD, 1998), Ásia (SONG et al., 2003) e África. A exemplo do que ocorre em outros países, no Brasil, as regiões costeiras tropicais ou subtropicais registram considerável prevalência de dirofilariose canina (LABARTHE et al., 1997a; AHID et al., 1999; SUASSUANA et al., 2003; ARAUJO et al., 2003; COSTA et al., 2004; LIMA; AHID, 2004;
REIFUR et al., 2004). Em áreas distantes do litoral, a doença também se apresenta de maneira freqüente (FERREIRA et al., 1999; FERNANDES et al., 2000).

\section{VETORES POTENCIAIS DE Dirofilaria immitis}

De acordo com Otto (1969), o desenvolvimento normal e completo das microfilárias até o estado infectante é completado apenas nos mosquitos da família Culicidae. Existem atualmente 72 espécies de culicídeos descritas como vetores de Dirofilaria immitis no mundo todo (LOZOVEI, 2001). Essas estão distribuídas entre os gêneros Culex, Aedes, Ochlerotatus, Anopheles, Mansonia, Psorophora e Coquillettidia.

No Brasil, as espécies Culex quinquefasciatus, Aedes taeniorbynchus, Aedes aegypti e Aedes scapularis foram consideradas como prováveis vetores na região sudeste (LOURENÇO-DE-OLIVEIRA; DEANE, 1995; MACEDO et al., 1998, SERRÃO et al., 2001). Enquanto que na região nordeste do país, Ahid; Lourenço-de-Oliveira (1999), Brito et al., (1999) e Lima; Ahid (2004) reportaram as espécies Aedes taeniorhynchus e Culex quinquefasciatus como vetores potenciais de Dirofilaria immitis.

\section{PREVENÇÃO E CONTROLE DA DIROFILARIOSE}

O risco de infecção por Dirofilaria immitis existe para animais e pessoas, desde que na área de domicílio seja introduzido algum animal microfilarêmico, o clima seja adequado e esses locais disponham de áreas de saneamento precário, sem redes adequadas de esgotos e tratamento de águas residuais, as quais podem servir para o desenvolvimento de uma ou mais espécies de mosquitos vetores de dirofilariose.

O diagnóstico da doença em cães pode ser confirmado pela presença de antígenos do parasita adulto na circulação por meio de técnica imunoenzimática - ELISA e pesquisa de microfilárias presentes no sangue de animais suspeitos, utilizando-se a técnica de KNOTT. O controle da doença implica no diagnóstico correto e tratamento dos animais afetados, rastreamento e identificação dos animais portadores, dos criadouros e das espécies de mosquitos vetores, conscientização da população a respeito dos perigos que a dirofilari- 
ose representa para o homem e animais e a profilaxia da doença por meio de educação sanitária. Tratamento curativo ou procedimento cirúrgico no cão portador de parasitas adultos deverá ser levado em consideração, pois animais não tratados poderão desenvolver quadro clínico cada vez mais graves, comprometendo drasticamente sua qualidade de vida. Entretanto, uma avaliação prévia da carga parasitária antes do tratamento com fármacos é necessária, uma vez que as dirofilárias adultas mortas podem desencadear quadro fatal de tromboembolismo.

De acordo com Labarthe (1997b.), em regiões onde os animais estão expostos à ação de mosquitos vetores, pode ser adotado tratamento microfilaricida preventivo com o devido acompanhamento pelo médico veterinário. Infelizmente, a utilização de medicações prescritas e administradas de maneira incorreta por leigos e práticos interferem de forma negativa, expondo o animal a risco de morte (LEITE et al., 2002). Também o custo financeiro dos procedimentos, além do risco da remoção cirúrgica das dirofilárias adultas, limita em algumas ocasiões a realização dessa prática pelo médico veterinário.

\section{Referências}

AHID, S. M. M.; LOURENÇO-DE-OLIVEIRA, R.; SARAIVA, L. Q. Dirofilariose canina na Ilha de São Luiz, Nordeste do Brasil: uma zoonose potencial. Cadernos de Saúde Pública, v. 15, p. 405-412. 1999.

AHID, S. M. M.; LOURENÇO-DE-OLIVEIRA, R. Mosquitos vetores potenciais de dirofilariose canina na Região Nordeste do Brasil. Revista de Saúde Pública, v. 33, n. 6, p. 560-566. 1999.

ARAUJO, R. T. et al. Canine dirofilariasis in the region of Conceição Lagoon, Florianópolis, and the Military Police Kennel, São José, State of Santa Catarina, Brazil. Veterinary Parasitology, v. 113, n. 3-4, p.239-242. 2003.

ATKINS, C. E. et al. Heartworm in cats: 50 cases (1985-1997). Journal of The American Veterinary Medical Association, v. 217, n. 3. p. 355-358. 2000.
BRITO, A. C. et al. Development of Dirofilaria immitis (Leydi ) in Aedes aegypti (L.) and Culex quinquefasciatus (Say) from Maceió, Alagoas, Brazil. Memórias do Instituto Oswaldo Cruz, v. 94, n. 4, p. 575-576, 1999.

BROWNE, L. E. et al. Pulmonary arterial disease in cats seropositive for Dirofilaria immitis but lacking adult heartworms in the heart and lungs. American Journal of Veterinary Research, v. 66, n. 9, p. 1544-1549, 2005.

CARLSON, B. L.; NIELSEN, S. W. Dirofilaria immitis infection in a gray fox. Journal of the American Veterinary Medical Association, v.183, n. 11, p. 1. 1983.

COSTA, R. C. et al. An update survey of the prevalence of canine dirofilariasis in a focus área of the city of Rio de Janeiro, Brazil. Revista Brasileira de Parasitologia Veterinária, v. 13, n. 1, p. 23-28, 2004 .

ETTINGER, S.J.; FELDMAN, E. C. Tratado de Medicina Veterinária. 5. ed. Rio de Janeiro, RJ: Guanabara Koogan, v. 1, p. 1038, 2004.

FERNANDES, C. G. N. et al. Aspectos epidemiológicos da dirofilariose canina no perímetro urbano de Cuiabá, Mato Grosso: emprego do "Immunoblot" e do teste de Knott modificado.

Brazilian Journal Veterinary Research Animal Science, v. 37, n. 6, p. 1-10, 2000.

FERREIRA, A. F.; BARBOSA, F. C.; MASTRANTONIO, E. C. Ocorrência da dirofilariose canina na cidade de Uberlândia, MG, Brasil. Veterinária Notícias, Uberlândia. v. 5, n. 1, p. 57-61, 1999.

FOIL, L.; ORIEL, T. C. Dirofilaria immitis (Leidy, 1856) in the Beaver, Castor canadensis. Journal of Parasitology, v. 61, n. 3, p. 433, 1975.

FORRESTER, D. J. et al. Heartworms in captive California Sea Lions. Journal of the American Veterinary Medical Association, v. 163, n. 6, p. 568-570, 1973.

FRANSON, J. C.; JORGENSON, R. D.; BOGESS, E. K. Dirofilariasis in Iowa coyotes. Journal of Wildlife Diseases, v. 12, n. 2, p. 165-166, 1976.

GOODFELOW, M.; SHAW, S. Exotic diseases in dogs and cats at risk of importation to Ireland. Irish Veterinary Journal, v. 58, n. 5, p. 271-277, 2005. 
GORTAZAR, C. et al. Factors affecting Dirofilaria immitis prevalence in red foxes in northeastern Spain. Journal of Wildlife Diseases, v. 30, n. 4, p. 545-547, 1994.

GUPTA, M. K. et al. Ocurrence of dirofilariosis in lions and tigers. Journal of Veterinary Parasitology, v. 13, n. 1, p. 77-78, 1999.

HAMIR, A. N.. Heartworm (Dirofilaria immitis) in the brain of a dog. Veterinary Record, v. 28, n. 9, p. 207-208, 1987.

HARWELL, G.; CRAIG, T.M. Dirofilariasis in a Red Panda. Journal of American Veterinary Medical Association, v. 179, n. 11, p. 1258. 1981.

HAYASAKI, M., et al. Diurnal variation in microfilaremia in a cat experimentally infected with larvae of Dirofilaria immitis. Veterinary Parasitology, v. 111, p. 267-271, 2003.

HIDAKA, Y. et al. Three dogs under 2 years of age with Heartworm Caval Syndrome. Journal of Veterinary Medicine Science, v. 65, n. 10, p. 1147-1149, 2003.

JOHNSON, C. A. III. Ursus americanus (Black Bear) a new host for Dirofilaria immitis. The Journal of Parasitology, v. 61, n. 5, p. 940, 1975.

JUNKUM, A. et al. Comparative studies on the biology and filarial susceptibility of selected bloodfeeding and autogenous Aedes togoi sub-colonies. Memórias do Instituto Oswaldo Cruz, v. 98, n. 4, p. 481-485, 2003.

KANG, S. et al. A harbor seal infection with Dirofilaria. Journal of Veterinary Clinic, v.19, n. 1, p. 92-94, 2002.

KASAI, N.; MALTOS, E. A.; COSTA, J. O. Dirofilaria immitis e Dipetalonema reconditum em cães de Vitória, Espírito Santo. Arquivos da Escola de Veterinária da UFMG. v. 33, n. 3, p. 425-429, 1981.

KELLY, J. D. Detection and differentiation of microfilariae in canine blood. Australian Veterinary Journal, v. 49, p. 23-27, 1973.

KLEIN, J. B.; STODDARD, E. D. Dirofilaria immitis recovered from a horse. American Veterinary Medical Association, v. 171, n. 4, p. 353-355, 1977.
KOTANI, T.; POWERS, K. G. Developmental stages of Dirofilaria immitis in the dog. American Journal Veterinary Research, v. 43, n.12, p. 2199-2206, 1982.

KIM, M. K. et al. The first human case of hepatic dirofilariasis. Journal of Korean Medical Science, v. 5, p. 686-690, 2002.

LABARTHE, N. et al. Description of the ocurrence of canine dirofilariasis in the State of Rio de Janeiro, Brazil. Memórias do Instituto Oswaldo Cruz, v. 92, n.1, p. 47-51, 1997a.

LABARTHE, N. V. Dirofilariose canina: diagnóstico, prevenção e tratamento adulticida. Revisão de Literatura. Clínica Veterinária, v. 10, p. 10-16, 1997b.

LEITE, L. C. Et al. Prescrição de medicamentos veterinários por balconistas e proprietários leigos de 26 estabelecimentos de comércio de animais em Curitiba-Pr-Brasil. In: SEMINÁRIO DE INICIAÇÃO CIENTÍFICA, 10., Curitiba, 2002. MOSTRA DE PESQUISA DA PUCPR, 4. 2002, Curitiba . Resumos... Curitiba , 2002.

LIMA, P. M.; AHID, S. M. M. Vetor natural da Dirofilaria immitis (nematoda) em Mossoró-RN. Caatinga, v.17, n. 2, p. 109-114, 2004.

LINDSEY, J. R. Identification of Canine Microfilariae. The Journal of American Veterinary Medicine Association, v. 146, n. 10, p. 1106-1114, 1965.

LOFTIN, K. M. et al. Potencial mosquito vectors of Dirofilaria immitis in Bernalillo County, New Mexico. Journal of American Mosquito Control Association, v. 11, n. 1, p. 90-93, 1995.

LOURENÇO-DE-OLIVEIRA, R.; DEANE, L. M. Presumed Dirofilaria immitis infections in wildcaught Aedes taeniorbynchus and Aedes scapularis in Rio de Janeiro, Brasil. Memórias do Instituto Oswaldo Cruz. v. 90, p. 387-388, 1995.

LOZOVEI, A. L. Culicídeos (Mosquitos) In: Carlos Brisola Marcondes. Entomologia Médica e Veterinária. São Paulo, SP: Atheneu, 2001. p. 59103.

LUDLAM, K. W.; JACHOWSKI, L. A.; OTTO, G. F. Potential vectors of Dirofilaria immitis. Journal of American Veterinary Medicine Association, v. 157, n. 1-12, p. 1354-1359, 1970. 
MACEDO, F. C.; LABARTHE, N.; LOURENÇODE-OLIVEIRA, R. Susceptibility of Aedes scapularis (Rondani, 1848) to Dirofilaria immitis (Leidy, 1856), an emerging zoonosis. Memórias do Instituto Oswaldo Cruz, v. 93, n. 4, p. 435-437, 1998.

MANTOVANI, A.; JACKSON, R. F. Transplacental transmission of microfilariae of Dirofilaria immitis in the Dog. The Journal of Parasitology, v. 52, n. 1, p.116, 1966.

MARANHO, A; NASCIMENTO, C. C.; SARTORI, F. I. 1997. Infecção por Dirofilaria immitis (Leydi, 1856) em lobo-marinho sulamericano (Arctocephalus australis - Zimmerman, 1783). In: CONGRESSO, 1., Pirassununga, 1997. VI ENCONTRO DA ASSOCIAÇÃO BRASILEIRA DE VETERINÁRIOS DE ANIMAIS SELVAGENS - ABRAVAS, 6., 1997, Pirassununga. Anais... São Paulo, SP: p. 21. 1997.

MARKS, C. A.; BLOOMFIELD, T. E. Canine heartworm (Dirofilaria immitis) detected in red foxes (Vulpes vulpes) in urban Melbourne. Veterinary Parasitology, v. 78, n. 2, p. 147-154, 1998.

MATSUDA, K.; BAEK, B. K.; LIM, C. W. Eurasian otter (Lutra lutra) a definitive host for Dirofilaria immitis. Journal of Zoo and Wildlife Medicine, v. 34, n. 2, p. 202-205, 2003.

McCALL, J. W. et al. Recent advances in heartworm disease. Veterinary Parasitology, v. 125, p. 105130, 2004.

MILANEZ DE CAMPOS, J. R. et al. Human Pulmonary Dirofilariasis: Analysis of 24 cases from São Paulo, Brazil. Chest. v. 112, n. 3, p. 729-733. 1997.

NAKAGAKI, K. et al. Dirofilaria immitis: experimental infection of rabits with immature fifth-stage worms. American Journal of Tropical Medicine \& Hygiene, v. 57, n. 6, p. 667-671, 1997.

NARINE, K. et al. Pulmonary presentation of Dirofilaria immitis (canine heartworm) in man. European Journal of Cardio- Thoracic Surgery, v. 16, n. 4, p. 475-477, 1999.

NEWTON, W. L.; WRIGHT, W. H. The occurrence of a Dog Filariid other than Dirofilaria immitis in the United States. Journal of Parasitology, v. 42, p. 246-256, 1956.
ORIHEL, T. C. Morphology of the larval stages of Dirofilaria immitis in the dog. The Journal of Parasitology, v. 47, p. 251-262, 1961

OSBORNE, T. C. Worms found in the heart and bloodvessels of a dog; symptoms of hydrophobia. The Western Journal of Medicine and Surgery, 1847.

OSHIRO, Y. et al. Pulmonary dirofilariasis: computer tomography findings and correlation with pathologic features. Journal of Computer Assisted Tomography, v. 28, n. 6, p. 796-800, 2004.

OTTO, G. F. Geographical distribuition, vectors, and life cicle of Dirofilaria immitis. Journal of American Veterinary Medical Association, v. 154, n. 4, p. 370-373, 1969.

PAES-de-ALMEIDA, E. C. et al. Kidney ultrastructural lesions in dogs experimentally infected with Dirofilaria immitis (Leidy, 1856). Veterinary Parasitology, v. 113, p. 57-168, 2003.

PENCE, D. B.; TEWES, M. E.; LAACK, L. L. Helminths of the Ocelot from Southern Texas. Journal of Wildlife Diseases, v. 39, n. 3, p. 683-689, 2003.

PINTO, C.; LUZ, A. Dirofilaria immitis na vesicula biliar de Canisfamiliaris. O Campo, v. 7, p. 36-37, 1936.

POLIZOPOULOU, Z. S. et al. Clinical and laboratory observations in 91 dogs infected with Dirofilaria immitis in Northern Greece. The Veterinary Record, v. 15, p. 466-469, 2000.

REIFUR, L.; THOMAZ-SOCCOL, V.; MONTIANIFERREIRA. Epidemiological aspects of filariosis on the coast of Paraná state, Brazil: with emphasis on Dirofilaria immitis. Veterinary Parasitology, v. 122, p. 273-286, 2004.

RODRIGUES-SILVA, R. et al. Dirofilaríase pulmonar humana no Estado do Rio de Janeiro, Brasil: relato de um caso. Revista da Sociedade Brasileira de Medicina Tropical, v. 37, n.1, p. 56-59, 2004.

RODRIGUEZ, B.; ROS-ALVAREZ, T.; GRANT, S.; ORIHEL, T. C. Human dirofilariasis in Costa Rica: Dirofilaria immitis in periorbital tissues. Parasite, v. 10, n. 1, p. 87-89, 2003.

RUSSEL, R. C. Report of field study on mosquito (Diptera: Culicidae) vectors of dog heartworm, Dirofilaria immitis Leydi (Spirurida: Onchocercidae) 
near Sydnei, N.S.W., and the implications for veterinary and public health concern. Australian Journal of Zoology, v. 33, n. 4, p. 461-472, 1985.

SASAI, H. et al. Echocardiografic diagnosis of dirofilariasis in a ferret. Journal of Small Animal Practice, v. 41, n. 4, p. 172-174, 2000.

SCHREY, C. F., TRAUTVETTER, E. Dirofilariosis canina y felina-diagnóstico e tratamiento. Waltham Focus, v. 8, n. 2, p. 23-31, 1998.

SEGOVIA, J. M. et al. Helminths in the wolf, Canis lupus, from north-western Spain. Journal of Helminthology, v. 75, n. 2, p. 183-192, 2001.

SERRÃO, M. L.; LABARTHE, N.; LOURENÇO-DEOLIVEIRA, R. Vectorial competence of Aedes aegypti (Linnaeus, 1762) Rio de Janeiro Strain, to Dirofilaria immitis (Leidy, 1856). Memórias do Instituto Oswaldo Cruz, v. 96, n. 5, p. 593-598, 2001.

SHAH, M. K. Human pulmonary dirofilariasis: review of the literature. Southern Medical Journal, v. 92, n. 3, p. 276-279, 1999.

SILVA ARAUJO, A Filaria immitis e a Filaria sanguinolenta no Brasil. Gazeta Médica da Bahia, v. 7, p. 295-312, 1878.

SNYDER, D. E. et al. Dirofilaria immitis in a racoon (Procyon lotor). Journal of Wildlife Diseases, $\mathrm{v}$. 25, n. 1, p. 130-131, 1989a.

SNYDER, D. E. et al Dirofilaria immitis in a river otter (Lutra canadensis) from Louisiana. Journal of Wildlife Diseases, v. 25, n. 4, p. 629, 1989 b.

SONG, K. H.; LEE, S. E. et al. Seroprevalence of canine dirofilariosis in South Korea. Veterinary Parasitology, v. 114, p. 231-236, 2003.

STARR, T. W.; MULLEY, R. C. Dirofilaria immitis in the dingo (Canis familiaris dingo) in a tropical regionof the northern territory, Australia. Journal of Wildlife Diseases, v. 24, n. 1, p. 164-165, 1988.

STRICKLAND, K.N. Canine and feline caval syndrome. Clinical Technical Small, Animal Practice, v. 13, n. 2, p. 88-95, 1998.
SUASSUANA, A. C. D.; de PAULA, V. V.; FEIJÓ, F. M. C. Ocorrência de cães parasitados por Dirofilaria immitis em Mossoró - RN. Revista Brasileira de Medicina Veterinária, v. 25, n. 5/6, p. 210-213, 2003.

TADA, I.; SAKAGUCHI, Y.; ETO, K. Dirofilaria in the abdominal cavity of a man in Japan. American Journal of Tropical Medicine \& Hygiene, v. 28, n. 6, p. 988-990, 1997.

TAKEUCHI, T. et al. Dirofilaria immitis infection in man: report of case of the infection in heart and inferior vena cava from Japan. American Journal of Tropical Medicine \& Hygiene, v. 305, n. 5, p. 966-969, 1981.

THEIS, J.H. et al. Case report: unusual location of Dirofilaria immitis in a 28-year-old man necessitates orchiectomy. American Journal of Tropical Medicine and Hygiene, v. 64, n. 5-6, p. 317-322, 2001.

THURMAN, J. D.; JOHNSON, B. J.; LICHTENFELS, J. R. Dirofilariasis with arteriosclerosis in a horse. Journal of American Veterinary Medical Association, v. 185, n. 5, p. 532-53, 1984.

TORRES, J.; FELIU, C. et al. Helminth parasites of the Eurasian Lutra lutra in southwest Europe. Journal of Helminthology, v. 78, p. 353-359, 2004 .

TRAVASSOS, L. P. Notas Helminthologicas. BrazilMedico, v. 35, n. 2-6, p. 67, 1921.

VITAL, R. J.; MATTOS, L. A.; MEIRELLES, G. S. P. Human pulmonary dirofilariasis: atypical presentation of a rare disease. Revista da Sociedade Brasileira de Medicina Tropical, v. 39, n. 1, p. 94-95, 2006.

WILLIAMS, J. F.; DADE, A. W. Dirofilaria immitis infection in a wolverine. The Journal of Parasitology, v. 62, n. 1, p. 174-175, 1976.

Recebido: 19/10/2005 Aprovado: 31/03/2006 